\title{
47. GEOCHEMICAL INVESTIGATIONS ON SEDIMENTS FROM THE MID-ATLANTIC RIDGE: LEG 37, DEEP SEA DRILLING PROJECT
}

\author{
D.S. Cronan, Department of Geology, Imperial College, London, SW 7
}

\section{INTRODUCTION}

Sediment samples from DSDP Leg 37 have been analyzed for a variety of elements. A particular aim of the investigation was to examine the lowermost sediments for evidence of basal metalliferous activity in the crestal area of the Mid-Atlantic Ridge, similar in nature to that previously reported on the East Pacific Rise (von der Borch and Rex, 1970; Cronan et al., 1972) and on or near the Central Indian Ocean Ridge (Cronan et al., 1974; Warner and Gieskes, 1974). A secondary objective was to evaluate possible gross compositional variations with depth in terms of changing sediment lithologies.

\section{ANALYTICAL TECHNIQUES}

All the samples were analyzed semiquantitatively by atomic absorption spectrophotometry for $\mathrm{Ca}, \mathrm{Fe}$, and $\mathrm{Mn}$, after total digestion in an $\mathrm{HNO}_{3}-\mathrm{HF}-\mathrm{HClO}_{4}$ mixture. Prior to analysis, the samples were air dried and ground to a fine powder. Element determinations were carried out using a Perkin-Elmer 403 AAS, and the results are presented in Table 1 .

\section{RESULTS}

All the samples are very rich in calcium carbonate, the lowest values (recalculated from $\mathrm{Ca}$ concentrations) being around $60 \%$.

With the possible exception of Site 333, there are no indications of increasing $\mathrm{Fe}$ and $\mathrm{Mn}$ concentrations in the sediments as basement is approached. Even at Site 333 evidence for such an increase is very limited.
It is apparent therefore that on the basis of the present data we have no real evidence in the Leg 37 sediments of metalliferous activity. However, this does not mean that such activity has not occurred, for the very high carbonate content of the sediments would make it difficult to detect. In order to evaluate this problem, it would be necessary to undertake some form of selective analysis of the sediments in order to remove the carbonate phases while leaving the remainder of the sediments as unaltered as possible.

\section{REFERENCES}

Cronan, D.S., van Andel, T.H., Heath, G.R., Dinkleman, M.G., Bennett, R.H., Bukry, D., Charleston, S., Kaneps, A., Rodolfo, K.S., and Yeats, R.S., 1972. Iron-rich basal sediments from the eastern equatorial Pacific: Leg 16, Deep Sea Drilling Project: Science, v. 175, p. 61-63.

Cronan, D.S., Damiani, V.V., Kinsman, D.J.J., and Thiede, J., 1974. Sediments from the Gulf of Aden and western Indian Ocean. In Fisher, R.L., Bunce, E.T., et al., Initial Reports of the Deep Sea Drilling Project, Volume 24: Washington (U.S. Government Printing Office), p. 10471110 .

von der Borch, C.C. and Rex, R.W., 1970. Amorphous iron oxide precipitates in sediments cored during Leg 5, Deep Sea Drilling Project. In McManus, D.A. et al., Initial Reports of the Deep Sea Drilling Project, Volume 5: Washington (U.S. Government Printing Office), p. 541544.

Warner, T.B. and Gieskes, J.M., 1974. Iron-rich basal sediments from the Indian Ocean: Site 245, Deep Sea Drilling Project. In Initial Reports of the Deep Sea Drilling Project, Volume 25: Washington (U.S. Government Printing Office), p. 385-403. 
TABLE 1

Chemical Analyses of Deep Sea Sediments from Leg 37, Deep Sea Drilling Project

\begin{tabular}{|c|c|c|c|c|c|c|c|c|c|c|c|c|c|c|c|c|}
\hline Hole & Core & Section & $\begin{array}{l}\text { Sampled } \\
(\mathrm{cm})\end{array}$ & $\begin{array}{l}\mathrm{Ca} \\
(\%)\end{array}$ & $\begin{array}{c}\mathrm{Al} \\
(\%)\end{array}$ & $\begin{array}{l}\mathrm{Fe} \\
(\%)\end{array}$ & $\begin{array}{c}\mathrm{Ni} \\
(\mathrm{ppm})\end{array}$ & $\begin{array}{c}\mathrm{Cd} \\
(\mathrm{ppm})\end{array}$ & $\begin{array}{c}\mathrm{Cu} \\
(\mathrm{ppm})\end{array}$ & $\begin{array}{c}\text { Co } \\
\text { (ppm) }\end{array}$ & $\begin{array}{c}\mathrm{Mn} \\
(\mathrm{ppm})\end{array}$ & $\begin{array}{c}\mathrm{Zn} \\
(\mathrm{ppm})\end{array}$ & $\begin{array}{c}\mathrm{Pb} \\
(\mathrm{ppm})\end{array}$ & $\begin{array}{c}\mathrm{Cr} \\
(\mathrm{ppm})\end{array}$ & $\begin{array}{c}\mathrm{Ti} \\
(\mathrm{ppm})\end{array}$ & $\begin{array}{l}\mathrm{Ng} \\
(\%)\end{array}$ \\
\hline $332 \mathrm{~A}$ & 4 & 4 & $90-92$ & 38.2 & 0.47 & 0.22 & 62 & 12 & 15 & 50 & 375 & 22 & 75 & 62 & 375 & 0.21 \\
\hline $332 \mathrm{~A}$ & 4 & 5 & $18-20$ & 37.3 & 0.47 & 0.20 & 75 & 11 & 20 & 50 & 387 & 21 & 75 & 62 & 250 & 0.22 \\
\hline $332 \mathrm{~A}$ & 5 & 3 & $116-118$ & 38.6 & 0.55 & 0.21 & 62 & 12 & 16 & 50 & 475 & 22 & 62 & 62 & 250 & 0.24 \\
\hline $332 \mathrm{~A}$ & 5 & 4 & $15-17$ & 37.0 & 0.63 & 0.48 & 75 & 11 & 15 & 50 & 450 & 22 & 62 & 62 & 625 & 0.27 \\
\hline $332 \mathrm{~A}$ & 5 & 5 & $13-15$ & 39.7 & 0.66 & 0.21 & 75 & 12 & 26 & 62 & 450 & 28 & 75 & 62 & 500 & 0.24 \\
\hline $332 \mathrm{~A}$ & 5 & 6 & 13-15 & 37.3 & 0.72 & 0.25 & 62 & 10 & 22 & 37 & 375 & 22 & 62 & 62 & 500 & 0.25 \\
\hline $332 \mathrm{~A}$ & 6 & 1 & $61-63$ & 38.1 & 0.81 & 0.47 & 75 & 12 & 17 & 62 & 500 & 27 & 62 & 112 & 625 & 0.27 \\
\hline $332 \mathrm{~A}$ & 6 & 2 & $12-14$ & 37.8 & 0.75 & 0.37 & 75 & 12 & 18 & 50 & 500 & 25 & 75 & 62 & 625 & 0.24 \\
\hline 332B & 1 & 1 & $24-26$ & 37.9 & 0.58 & 0.27 & 62 & 12 & 20 & 50 & 437 & 25 & 87 & 75 & 375 & 0.24 \\
\hline 332B & 1 & 2 & $17-19$ & 38.6 & 0.52 & 0.20 & 62 & 15 & 22 & 50 & 437 & 23 & 87 & 62 & 375 & 0.22 \\
\hline 332B & 1 & 3 & $18-20$ & 37.2 & 0.43 & 0.40 & 75 & 12 & 20 & 50 & 337 & 23 & 100 & 50 & 250 & 0.24 \\
\hline $332 \mathrm{~B}$ & 1 & 4 & $18-20$ & 37.4 & 0.67 & 0.32 & 62 & 11 & 17 & 50 & 375 & 25 & 62 & 62 & 500 & 0.24 \\
\hline 333 & 2 & 1 & $77-79$ & 37.7 & 0.53 & 0.28 & 62 & 15 & 16 & 50 & 550 & 22 & 62 & 62 & 500 & 0.20 \\
\hline 333 & 2 & 2 & $77-79$ & 38.6 & 0.55 & 0.20 & 75 & 12 & 16 & 50 & 475 & 22 & 62 & 62 & 375 & 0.19 \\
\hline 333 & 2 & 3 & $69-70$ & 38.6 & 0.38 & 0.12 & 62 & 12 & 18 & 37 & 512 & 22 & 87 & 75 & 250 & 0.21 \\
\hline 333 & 2 & 4 & $65-69$ & 37.8 & 1.16 & 0.27 & 75 & 12 & 20 & 50 & 425 & 30 & 75 & 62 & 625 & 0.24 \\
\hline 333 & 2 & 6 & $65-69$ & 38.3 & 0.35 & 0.12 & 62 & 12 & 18 & 50 & 325 & 20 & 87 & 62 & 500 & 0.17 \\
\hline 333 & 3 & 1 & $65-67$ & 38.6 & 0.43 & 0.46 & 75 & 12 & 18 & 50 & 412 & 27 & 62 & 100 & 500 & 0.20 \\
\hline 333 & 3 & 2 & $65-67$ & 39.2 & 1.07 & 0.36 & 62 & 12 & 17 & 50 & 412 & 20 & 75 & 37 & 375 & 0.21 \\
\hline 333 & 3 & 3 & $65-67$ & 37.3 & 0.85 & 0.33 & 62 & 15 & 21 & 50 & 375 & 26 & 100 & 75 & 500 & 0.25 \\
\hline 333 & 3 & 4 & $84-86$ & 38.6 & 0.42 & 0.18 & 75 & 12 & 22 & 50 & 412 & 25 & 87 & 62 & 250 & 0.23 \\
\hline 333 & 3 & 5 & $65-67$ & 37.3 & 0.53 & 0.26 & 75 & 10 & 18 & 50 & 487 & 18 & 87 & 75 & 375 & 0.24 \\
\hline 333 & 3 & 6 & $65-67$ & 38.3 & 0.47 & 0.23 & 62 & 12 & 17 & 50 & 437 & 18 & 87 & 50 & 250 & 0.23 \\
\hline 333 & 4 & 1 & $65-67$ & 39.7 & 0.47 & 0.20 & 62 & 13 & 13 & 50 & 375 & 20 & 75 & 62 & 500 & 0.22 \\
\hline 333 & 4 & 2 & $65-67$ & 39.7 & 0.61 & 0.17 & 62 & 10 & 17 & 50 & 312 & 22 & 87 & 75 & 500 & 0.22 . \\
\hline 333 & 4 & 3 & $65-67$ & 39.7 & 0.32 & 0.16 & 62 & 12 & 15 & 37 & 425 & 22 & 62 & 62 & 500 & 0.22 \\
\hline 333 & 4 & 4 & $65-67$ & 38.6 & 0.46 & 0.22 & 75 & 37 & 15 & 62 & 337 & 32 & 62 & 62 & 500 & 0.22 \\
\hline 333 & 4 & 5 & $65-67$ & 37.9 & 0.65 & 0.31 & 75 & 13 & 15 & 50 & 312 & 22 & 75 & 62 & 500 & 0.23 \\
\hline 333 & 5 & 2 & $65-67$ & 38.6 & 0.42 & 0.16 & 75 & 12 & 12 & 50 & 325 & 22 & 62 & 62 & 500 & 0.19 \\
\hline 333 & 5 & 4 & $65-67$ & 36.4 & 1.26 & 0.63 & 62 & 12 & 20 & 50 & 387 & 37 & 87 & 75 & 1000 & 0.43 \\
\hline 333 & 6 & 1 & $65-67$ & 39.2 & 0.47 & 0.21 & 75 & 12 & 15 & 50 & 387 & 22 & 75 & 50 & 375 & 0.23 \\
\hline 333 & 6 & 2 & $65-67$ & 37.0 & 0.98 & 0.47 & 75 & 15 & 18 & 50 & 337 & 27 & 75 & 50 & 875 & 0.34 \\
\hline 333 & 7 & 2 & $65-67$ & 38.8 & 0.91 & 0.47 & 62 & 10 & 22 & 62 & 662 & 27 & 75 & 75 & 875 & 0.34 \\
\hline 333 & 1 & 1 & $65-67$ & 38.5 & 0.45 & 0.22 & 75 & 11 & 15 & 50 & 375 & 22 & 75 & 62 & 250 & 0.21 \\
\hline 334 & 1 & 1 & $65-67$ & 38.6 & 1.10 & 0.61 & 62 & 12 & 30 & 50 & 712 & 30 & 225 & 62 & 750 & 0.28 \\
\hline 334 & 1 & 2 & $65-67$ & 36.8 & 1.05 & 0.58 & 75 & 12 & 32 & 50 & 725 & 27 & 62 & 62 & 875 & 0.30 \\
\hline 334 & 2 & 1 & $65-67$ & 38.3 & 0.40 & 0.21 & 75 & 12 & 12 & 50 & 200 & 22 & 87 & 62 & 250 & 0.19 \\
\hline 334 & 2 & 2 & $65-67$ & 37.8 & 0.68 & 0.36 & 75 & 13 & 15 & 62 & 237 & 25 & 100 & 62 & 875 & 0.21 \\
\hline 334 & 2 & 3 & $65-67$ & 38.1 & 0.81 & 0.45 & 75 & 11 & 126 & 50 & 225 & 30 & 75 & 62 & 1250 & 0.27 \\
\hline 334 & 2 & 4 & $65-67$ & 36.9 & 0.82 & 0.43 & 62 & 12 & 22 & 50 & 250 & 13 & 62 & 62 & 1000 & 0.25 \\
\hline 334 & 2 & 5 & $65-67$ & 37.6 & 0.77 & 0.37 & 75 & 13 & 18 & 62 & 237 & 21 & 62 & 62 & 1000 & 0.24 \\
\hline 334 & 2 & 6 & $65-67$ & 37.9 & 0.51 & 0.32 & 75 & 12 & 11 & 50 & 275 & 21 & 75 & 62 & 500 & 0.21 \\
\hline 334 & 3 & 2 & $65-67$ & 36.7 & 0.90 & 1.00 & 75 & 12 & 37 & 50 & 312 & 45 & 75 & 62 & 1000 & 0.36 \\
\hline 334 & 4 & 1 & $65-67$ & 34.2 & 1.26 & 1.17 & 62 & 11 & 20 & 50 & 387 & 31 & 87 & 75 & 2375 & 0.46 \\
\hline 334 & 5 & 3 & $65-67$ & 33.4 & 2.50 & 1.75 & 62 & 11 & 21 & 50 & 475 & 41 & 62 & 75 & 3125 & 0.68 \\
\hline 334 & 5 & 4 & $65-67$ & 25.7 & 5.66 & 3.99 & 62 & 11 & 40 & 62 & 762 & 61 & 62 & 100 & 7728 & 1.41 \\
\hline 334 & 6 & 1 & $65-67$ & 25.8 & 6.18 & 3.73 & 62 & 11 & 32 & 50 & 800 & 62 & 62 & 75 & 7728 & 1.23 \\
\hline 334 & 6 & 2 & $65-67$ & 30.7 & 3,22 & 2.07 & 62 & 11 & 25 & 50 & 700 & 43 & 75 & 75 & 3750 & 0.79 \\
\hline 334 & 7 & 1 & $65-67$ & 30.2 & 3.34 & 2.26 & 75 & 11 & 25 & 50 & 712 & 52 & 75 & 75 & 3750 & 0.81 \\
\hline 334 & 7 & 2 & $100-102$ & 31.1 & 2.96 & 2.30 & 62 & 11 & 22 & 50 & 687 & 46 & 62 & 62 & 3750 & 0.67 \\
\hline 334 & 7 & 3 & $24-26$ & 30.9 & 2.96 & 1.96 & 75 & 11 & 25 & 50 & 675 & 42 & 87 & 75 & 3375 & 0.65 \\
\hline 334 & 7 & 4 & $65-67$ & 30.9 & 3.22 & 2.25 & 75 & 11 & 21 & 50 & 750 & 40 & 75 & 75 & 3625 & 0.72 \\
\hline 334 & 7 & 5 & $65-67$ & 29.8 & 3.34 & 2.23 & 75 & 7 & 26 & 50 & 725 & 43 & 62 & 75 & 3750 & 0.75 \\
\hline 334 & 7 & 6 & $65-67$ & 29.7 & 3.34 & 2.18 & 62 & 11 & 27 & 50 & 725 & 45 & 75 & 75 & 6440 & 0.79 \\
\hline 334 & 8 & 1 & $115-117$ & 26.1 & 3.22 & 3.34 & 62 & 10 & 22 & 50 & 750 & 61 & 50 & 87 & 6440 & 0.104 \\
\hline 334 & 8 & 2 & $49-49$ & 26.1 & 5.02 & 3.47 & 62 & 10 & 30 & 50 & 700 & 60 & 75 & 75 & 6440 & 1.15 \\
\hline 334 & 9 & 2 & $111-113$ & 33.3 & 2.02 & 1.45 & 62 & 15 & 22 & 50 & 600 & 36 & 100 & 62 & 3125 & 0.51 \\
\hline 334 & 9 & 3 & $65-67$ & 32.8 & 2.30 & 1.50 & 75 & 13 & 25 & 50 & 600 & 36 & 75 & 62 & 3000 & 0.56 \\
\hline 334 & 9 & 4 & $60-62$ & 33.3 & 2.16 & 1.50 & 62 & 12 & 20 & 50 & 600 & 37 & 75 & 62 & 3000 & 0.55 \\
\hline 334 & 11 & 2 & $64-66$ & 36.7 & 0.96 & 0.57 & 62 & 12 & 15 & so & 425 & 27 & 62 & 62 & 1250 & 0.30 \\
\hline 334 & 11 & 3 & $65-67$ & 36.0 & 0.88 & 0.75 & 75 & 13 & 13 & 50 & 512 & 26 & 75 & 50 & 1000 & 0.30 \\
\hline 334 & 11 & 4 & $65-67$ & 37.8 & 0.77 & 0.52 & 62 & 10 & 15 & 50 & 625 & 22 & 75 & 62 & 1000 & 0.27 \\
\hline 334 & 12 & 2 & $65-67$ & 35.8 & 1.01 & 0.68 & 75 & 12 & 15 & 50 & 512 & 30 & 62 & 62 & 1125 & 0.32 \\
\hline 334 & 12 & 3 & $65-67$ & 36.3 & 1.25 & 0.87 & 62 & 12 & 17 & 50 & 575 & 30 & 75 & 62 & 1625 & 0.39 \\
\hline 334 & 12 & 4 & $65-67$ & 36.9 & 1.27 & 0.90 & 75 & 11 & 20 & 50 & 650 & 30 & 75 & 75 & 1250 & 0.40 \\
\hline 334 & 13 & 2 & $108-110$ & 36.7 & 1.12 & 0.78 & 62 & 12 & 18 & 50 & 700 & 27 & 62 & 75 & 1500 & 0.36 \\
\hline 334 & 13 & 5 & $65-67$ & 35.8 & 1.13 & 0.83 & 62 & 11 & 13 & 50 & 575 & 26 & 62 & 62 & 1250 & 0.37 \\
\hline 334 & 13 & 6 & $65-67$ & 36.9 & 1.05 & 0.72 & 75 & 12 & 13 & 50 & 575 & 30 & 62 & 62 & 1125 & 0.36 \\
\hline 334 & 14 & 1 & $88-90$ & 36.8 & 0.98 & 0.75 & 75 & 11 & 17 & 50 & 762 & 27 & 87 & 75 & 1000 & 0.36 \\
\hline 335 & 1 & 2 & $65-67$ & 35.4 & 2.15 & 0.56 & 75 & 12 & 25 & 50 & 237 & 26 & 62 & 75 & 750 & 0.35 \\
\hline 335 & 1 & 3 & $65-67$ & 38.3 & 0.91 & 0.32 & 75 & 12 & 16 & 50 & 212 & 24 & 75 & 50 & 500 & 0.23 \\
\hline 335 & 1 & 4 & $65-67$ & 37.4 & 1.05 & 0.32 & 75 & 10 & 14 & 50 & 462 & 27 & 75 & 62 & 500 & 0.24 \\
\hline 335 & 2 & 2 & 65-67 & 38.7 & 0.50 & 0.16 & 75 & 12 & 12 & 50 & 612 & 21 & 87 & 50 & 250 & 0.18 \\
\hline 335 & 2 & 3 & $65-67$ & 39.6 & 0.51 & 0.16 & 62 & 15 & 18 & 50 & 550 & 25 & 75 & 50 & 250 & 0.19 \\
\hline 335 & 2 & 4 & $65-67$ & 39.7 & 0.38 & 0.16 & 75 & 12 & 12 & 50 & 300 & 20 & 75 & 62 & 500 & 0.17 \\
\hline 335 & 2 & 5 & $65-67$ & 39.2 & 0.35 & 0.13 & 75 & 12 & 16 & 50 & 300 & 20 & 100 & 75 & 375 & 0.17 \\
\hline 335 & 3 & 1 & $65-67$ & 38,8 & 0.43 & 0.17 & 62 & 14 & 15 & 50 & 350 & 20 & 75 & 62 & 375 & 0.18 \\
\hline 335 & 4 & 2 & $65-67$ & 39.5 & 0.32 & 0.12 & 62 & 12 & 12 & 50 & 237 & 20 & 75 & 62 & 250 & 0.17 \\
\hline 335 & 4 & 3 & $65-67$ & 38.6 & 0.38 & 9.13 & 62 & 12 & 15 & 50 & 275 & 20 & 75 & 50 & 387 & 0.18 \\
\hline
\end{tabular}

